\title{
Cellular automaton modelling of lightning-induced and man made forest fires
}

\author{
R. Krenn and S. Hergarten \\ Institute of Earth Sciences, University of Graz, Heinrichstraße 26, 8010 Graz, Austria \\ Received: 8 April 2009 - Revised: 11 September 2009 - Accepted: 29 September 2009 - Published: 30 October 2009
}

\begin{abstract}
The impact of forest fires on nature and civilisation is conflicting: on one hand, they play an irreplaceable role in the natural regeneration process, but on the other hand, they come within the major natural hazards in many regions. Their frequency-area distributions show power-law behaviour with scaling exponents $\alpha$ in a quite narrow range, relating wildfire research to the theoretical framework of self-organised criticality. Examples of self-organised critical behaviour can be found in computer simulations of simple cellular automaton models. The established self-organised critical Drossel-Schwabl forest fire model is one of the most widespread models in this context. Despite its qualitative agreement with event-size statistics from nature, its applicability is still questioned. Apart from general concerns that the Drossel-Schwabl model apparently oversimplifies the complex nature of forest dynamics, it significantly overestimates the frequency of large fires. We present a modification of the model rules that distinguishes between lightning-induced and man made forest fires and enables a systematic increase of the scaling exponent $\alpha$ by approximately $1 / 3$. In addition, combined simulations using both the original and the modified model rules predict a dependence of the overall event-size distribution on the ratio of lightning induced and man made fires as well as a splitting of their partial distributions. Lightning is identified as the dominant mechanism in the regime of the largest fires. The results are confirmed by the analysis of the Canadian Large Fire Database and suggest that lightning-induced and man made forest fires cannot be treated separately in wildfire modelling, hazard assessment and forest management.
\end{abstract}

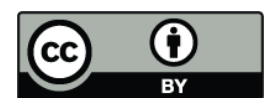

Correspondence to: R. Krenn (roland.krenn@uni-graz.at)

\section{Introduction}

During the last decades, extensive event-size statistics for several natural hazards have become available. In many cases, e.g., earthquakes (Gutenberg and Richter, 1954), landslides (Hovius et al., 1997; Malamud et al., 2004), rockfalls (Malamud et al., 2004), and the subject of this paper, forest fires (e.g., Minnich and Chou, 1997; Ricotta et al., 1999; Malamud et al., 1998, 2005; Ricotta et al., 2001; Song et al., 2001), power-law distributions have been found. This means that the probability density $p(s)$ related to the event size $s$ decreases like $s^{-\alpha}$, with $\alpha$ typically called the scaling exponent. Despite many exogenous factors influencing wildfire occurrence like fuel accumulation, topography, and weather (Pyne et al., 1996), vegetation types (Minnich, 1983), different fire protection management policies (Minnich, 1983, 2001; Minnich and Chou, 1997), or anthropogenic effects (Cardille et al., 2001; Malamud et al., 2005), it was found that, under a broad range of spatial and temporal conditions, forest fires exhibit power-law behaviour on their frequencysize distributions over about two to five decades of wildfire area, with a majority of the scaling exponents $\alpha$ falling into a range $1.1 \leq \alpha \leq 1.9$.

The similar event-size statistics of such obviously different phenomena suggest a unifying concept on a more fundamental level. On this note, self-organised criticality (e.g., Bak et al., 1987; Bak, 1996; Jensen, 1998; Hergarten, 2002) has become a magic word to explain power-law distributed events in dynamical systems. The first comprehensive study relating wildfire research to the theoretical framework of self-organised criticality was published by Malamud et al. (1998).

Examples of self-organised critical behaviour can be found in computer simulations of simple cellular automaton models. In connection with forest fires, the automaton commonly referred to as the forest fire model developed by Drossel and Schwabl (1992) (DS-FFM in the following) is one of the

Published by Copernicus Publications on behalf of the European Geosciences Union. 
most widespread models in this context. It is usually studied on a regular square lattice with periodic boundary conditions, where each site can be either occupied by a model tree or empty. In each time step, new trees are planted with a probability $p$ on empty sites. Each tree is subsequently ignited with a lightning probability $f$, which then burns down the entire geometric cluster of trees connected to it via nearestneighbour relations. In most cases, the von Neumann neighbourhood with four adjacent cells is considered. The size $s$ of a fire is measured in terms of the number of burnt trees. In the limit of small time steps, i.e., $p \rightarrow 0$ and $f \rightarrow 0$, the growth rate $\theta=p / f$, which also sets the scale for the average fire size, becomes the only relevant parameter of the model. In each time step, the dynamics of the system is then determined according to the following update rules: (i) A total of $\theta$ sites is randomly chosen. If a site is empty, its state turns into occupied, otherwise nothing happens. (ii) A randomly chosen site is ignited. If this site is occupied by a tree, the entire cluster of trees connected to it burns down. Simulation results indicated critical behaviour in the limit $\theta \rightarrow \infty$ (Drossel and Schwabl, 1992; Clar et al., 1994). However, the scaling behaviour is not simple, and there has been much discussion whether the DS-FFM is self-organised critical in the strict sense or not (Schenk et al., 2002; Grassberger, 2002; Pruessner and Jensen, 2002).

Despite the qualitative agreement with fire size statistics from nature, the applicability of the DS-FFM remains still questioned. Apart from general concerns that the DSFFM apparently oversimplifies the complex nature of forest dynamics (Caldarelli et al., 2001), it significantly overestimates the frequency of large fires: since differences in the power-law exponent become more important as $\alpha$ comes close to one, the quantitative agreement between the DSFFM $(1.0 \leq \alpha \leq 1.25)$ and nature $(1.1 \leq \alpha \leq 1.9)$ is in fact rather weak: the cumulative distribution $P(s)$ decays like $s^{-(\alpha-1)}$ (Hergarten, 2002), and thus changes strongly if $\alpha$ tends to one. To overcome this problem is not trivial because a systematic variation of the power-law exponent $\alpha$ is not provided by the model. There were many attempts to modify the model rules towards being more realistic with respect to the propagation of fires, e.g., by introducing immune sites (Drossel and Schwabl, 1993; Clar et al., 1994), facilitating propagation by allowing diagonal connections or introducing anisotropic propagation (Hergarten, 2002), but none of them improved the results substantially.

\section{Accessible perimeter ignition forest fire model (AP-FFM)}

We found that modifying the rule of ignition has a stronger effect than changing the rules of propagation. In the DSFFM and its derivatives, each site naturally has, metaphorically speaking, equal susceptibility to be struck by lightning. But if we consider highways, roads, railways, trails or glades and urban areas and take human action into account, ignition should occur preferably at the border of a forest or at locations where the forest is not very dense (cf., Cardille et al., 2001; Stephens and Ruth, 2005; Maingi and Henry, 2007). An obvious choice would be to modify the model rules in such a way that ignition takes place at empty sites instead of occupied sites, and that fire propagates from an empty ignition site to those of its four nearest neighbours which are occupied by trees. However, this modification would suffer from the problem that clusters of trees in the DS-FFM closely resemble two-dimensional site-percolation clusters at or above the critical concentration $p_{c}$. This implies that the total number of perimeter sites $N_{p}$ scales in the same way as the total cluster size $s, N_{p} \sim s$ (Kunz and Souillard, 1978). Thus such a modification affects the frequency of small fires, but its effect on the frequency of large fires is negligible.

We therefore restricted the admissible ignition sites to those being part of the accessible perimeter of a cluster. The concept of the accessible perimeter was introduced by Grossman and Aharony (1986). The accessible perimeter of a fractal object consists of those perimeter sites which can be reached, in principle, by a random walker coming from infinity. Figure 1 illustrates the basic idea: fires start from sites that are commonly understood as the edge of a forest. This approach is further supported by the result that the fractal dimension of the accessible perimeter found for site percolation as well as in our model simulations coincides with the fractal dimension of the accessible perimeter of real forest fires (Caldarelli et al., 2001).

Consequently, this leads to a modified set of rules for updating the lattice in each time step: while the first part remains unchanged, the second rule turns into: (ii) A randomly chosen site is ignited. If this site is an (empty) accessible perimeter site, the entire cluster of trees connected to it burns down.

\section{Results and discussion}

In Fig. 2 we compare the results of this accessible perimeter ignition forest fire model (AP-FFM) with those of the original DS-FFM. The behaviour of the curves remains qualitatively the same, i.e., fires are still distributed according to power laws, but the exponent $\alpha$ increases significantly. While we obtained $\alpha=1.22$ for the DS-FFM, our estimate for the AP-FFM is $\alpha=1.51$. We found that the probability density of the present clusters $n(s)$ behaves quite similarly in both models, so this finding can be understood by considering the probability of a cluster of size $s$ to be ignited. The total number of accessible perimeter sites $N_{a p}$ scales with the radius of gyration $r$ as

$N_{a p} \sim r^{d_{a p}}$,

with an associated fractal dimension $d_{a p}$. The latter scales like $s^{1 / d_{f}}$, where $d_{f}$ is the fractal dimension of 


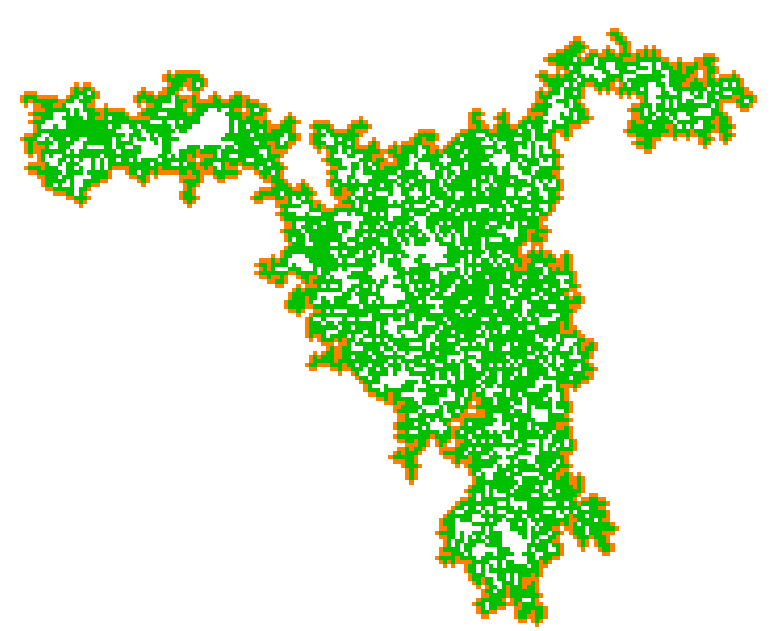

Fig. 1. Illustration of a cluster of trees (green) and its accessible perimeter (orange). The total number of accessible perimeter sites $N_{a p}$ scales with the cluster size $s$ approximately as $N_{a p} \sim s^{2 / 3}$.

two-dimensional percolation clusters, so that

$N_{a p} \sim s^{\frac{d_{a p}}{d_{f}}}$.

For $d=2$ site-percolation, $d_{f}=91 / 48$ at $p_{c}$ and $d_{f}=2$ above $p_{c}$, which applies to the large clusters in both models. In combination with $d_{a p} \approx 4 / 3$ (Grossman and Aharony, 1986), we obtain

$N_{a p} \sim s^{\frac{2}{3}}$.

Since the probability of a cluster of size $s$ to be ignited is proportional to $s$ in the DS-FFM and to $s^{2 / 3}$ in the AP-FFM, these probability densities scale with

$p_{\text {DS }}(s) \sim n(s) s$,

$p_{\mathrm{AP}}(s) \sim n(s) s^{\frac{2}{3}}$,

so that

$p_{\mathrm{AP}}(s) \sim p_{\mathrm{DS}}(s) s^{-\frac{1}{3}} \sim s^{-\left(\alpha+\frac{1}{3}\right)}$.

Therefore the power-law exponent of the AP-FFM is approximately $1 / 3$ greater than that of the DS-FFM, and the faster decay of the probability density with the event size in the AP-FFM can be immediately derived from the lower ignition probability of large clusters.

With regard to fires in nature, it is unlikely that they are triggered by a single ignition mechanism. However, we found that fire statistics of a region where both lightning and human action are present cannot be predicted by simulating both cases separately and just superposing the statistics. In order to illustrate this, we performed combined simulations including both "lightning" (DS-FFM) and "human action" (AP-FFM).

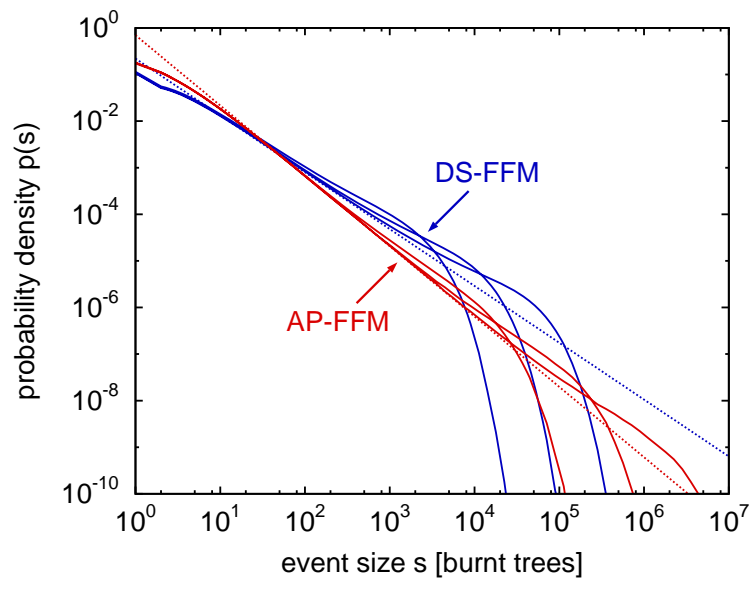

Fig. 2. Binned simulated event-size distributions for the original DS-FFM (blue) and our modified AP-FFM (red). Curves correspond to growth rates $\theta=256,1024$ and 4096 from left to right. The dotted lines indicate the slope for intermediate event sizes estimated by extrapolation to $\theta \rightarrow \infty$.

In Fig. 3, we compare the results for the overall eventsize distribution $p(s)$ for different ratios of lightning-induced and man made fires with that of the DS-FFM and the APFFM. We estimate $\alpha=1.42$ for a percentage ratio 25/75 (lightning/man made), $\alpha=1.36$ for $50 / 50$, and $\alpha=1.30$ for $75 / 25$, compared to $\alpha=1.51$ for $0 / 100$ (AP-FFM) and $\alpha=1.22$ for $100 / 0$ (DS-FFM). Hence, the scaling exponent $\alpha$ decreases systematically with lightning rate by approximately $1 / 3$. In addition, the long tail of the distribution occurring in the APFFM vanishes with increasing percentage rate of lightningcaused fires, so that the frequency of the largest fires is much lower than predicted from a simple superposition of the DSFFM and the AP-FFM.

The deviation of the graphs in Fig. 3 at large event sizes indicates an interdependence of lightning-induced and man made in the combined simulations. Figure 4 shows the partial lightning-induced and man made event-size distributions for percentage ratios 75/25, 50/50 and 25/75 (lightning/man made) in comparison with the overall distributions of the DSFFM (100/0) and the AP-FFM (0/100). For intermediate event sizes, the scaling exponents $\alpha$ of the respective probability densities still persist. As a result, this ensures that the assumption of two different ignition mechanisms can be easily proved by analysing the particular distributions of natural fire statistics. Concentrating on the tails of the distributions, one observes that the frequency of the largest events is lower for the man made fires in the combined simulations. This indicates that the largest fires are almost lightning-induced if both ignition mechanisms are present. This arises from the probability of a cluster of size $s$ to be ignited. We obtain from Eqs. (4) and (5) that their ratio $p_{\mathrm{DS}}(s) / p_{\mathrm{AP}}(s)$ scales with the cluster size $s$ like $s^{1 / 3}$, what entails that in the combined simulations the largest clusters a mainly consumed by 


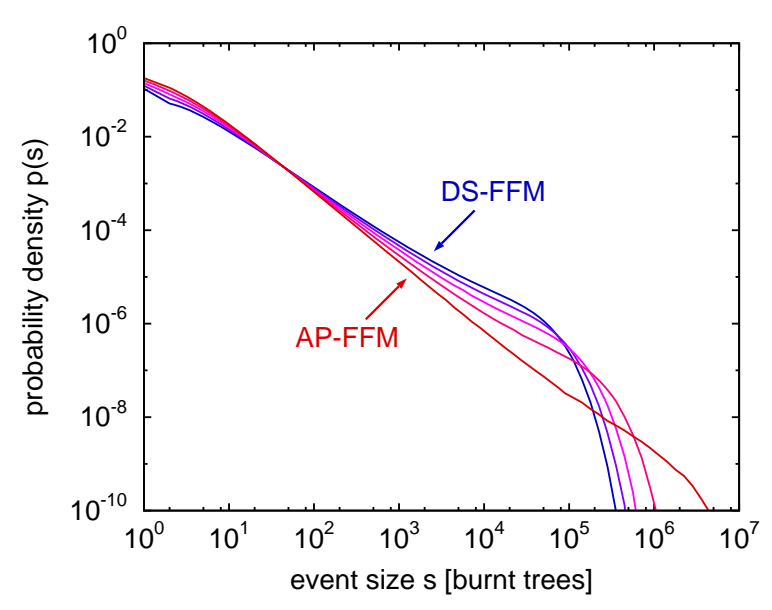

Fig. 3. Binned overall event-size distributions derived from combined simulations of lightning-induced and man made fires, percentage ratios 100/0 (DS-FFM, blue), 75/25 (indigo), 50/50 (magenta), 25/75 (carmine) and 0/100 (AP-FFM, red) from left to right, for $\theta=4096$.

lightning fires. Extremely large man made fires as occurring in the AP-FFM are therefore effectively suppressed and become less hazardous in a mixed environment, but only as long as lightning-induced fires avoid the occurrence of very large man made fires. In order to illustrate this quantitatively, we give some numbers on the top 0.1 percentiles from the combined 75/25 simulation (lightning/man made) and the AP-FFM (0/100) with identical growth rates $\theta=4096$ : in the combined $75 / 25$ simulation, $0.1 \%$ of the fires had a size of more than 210000 trees, whereby the man made fires accounted for only $2.6 \%$ of events (compared to $25 \%$ in total). In contrast, $0.1 \%$ of the (pure man made) fires in the APFFM are even bigger than 1700000 trees. In other words, the biggest out of 1000 events has become more than six times larger in absence of ignition by lightning. Of course, these values depend in general on both the growth rate $\theta$ and the ratio between lightning-induced and man made fires.

Conclusively, the combined simulations make the following predictions: (i) The scaling exponent of the overall event-size distribution $p(s)$ depends on the ratio of lightninginduced and man made fires. (ii) There is a difference in the exponents of lightning-induced and man made fires, with $\alpha_{\mathrm{LTG}} \approx \alpha_{\mathrm{DS}} \approx 1.22, \alpha_{\mathrm{MAN}} \approx \alpha_{\mathrm{AP}} \approx 1.51$, and $\Delta \alpha \approx 1 / 3$. (iii) At reasonable lightning rates, the largest fires are governed by lightning.

The Canadian Large Fire Database (LFDB) (Canadian Forest Service, 2002) provides an extensive data set to test these hypotheses. The database is available online and contains information on 11231 fires of more than $2 \mathrm{~km}^{2}$ in area for the 1959 to 1999 period and distinguishes between fires caused by lightning or human action. $72 \%$ (8086) of the LFDB fires were initiated by lightning, and 25\% (2754) by humans (the rest is unknown). A detailed analysis of the

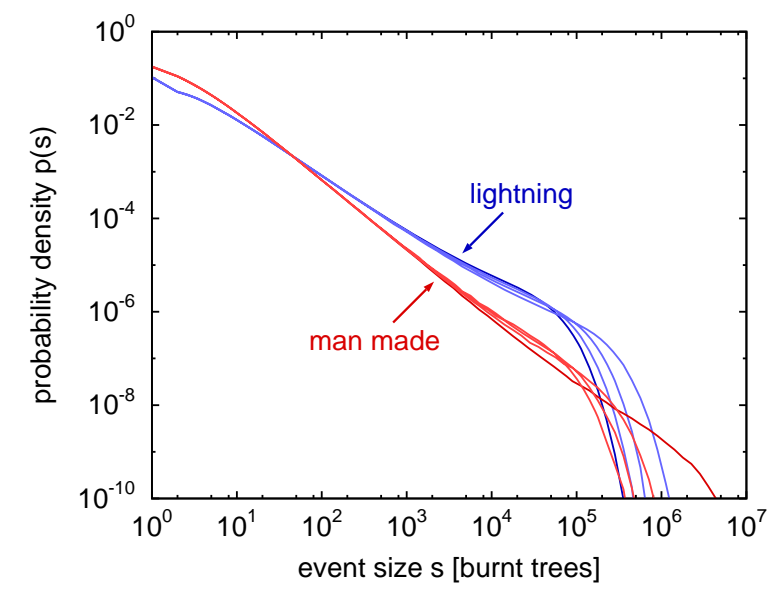

Fig. 4. Binned partial event-size distributions of lightning-induced (light blue) and man made fires (light red) for percentage ratios $75 / 25,50 / 50$ and 25/75 (lightning/man made) in comparison with the overall distributions of the DS-FFM (100/0, blue) and the APFFM (0/100, red), for $\theta=4096$.

LFDB data led to the result that the lightning-caused fires contributed to $85 \%$ of the total burnt area (Stocks et al., 2002), so they must be larger than those induced by humans in the mean.

Figure 5 shows the probability densities of fire sizes estimated from the LFDB data. The estimates were made using logarithmic binning with a factor $\sqrt{2}$ between the bins, corresponding to 6.6 bins per decade. Assuming that the deviation of the graphs at large event sizes is due to a cutoff as it occurs in the event-size statistics of most natural hazards, the overall distribution $p(s)$ follows a power law with an exponent $\alpha=1.30$. We note that this result is in complete quantitative agreement with the scaling exponent obtained from the comparable combined simulation where $75 \%$ of the fires were lightning-caused and 25\% man made. As conjectured, the LFDB data yield power-law distributions for both classes of fires separately. The power law of the man made fires has in fact a higher exponent, in agreement with the smaller average fire size found by Stocks et al. (2002). We estimate $\alpha_{\mathrm{LTG}}=1.20$ for ignition by lightning and $\alpha_{\mathrm{MAN}}=1.61$ for the man made fires, with a difference $\Delta \alpha=0.41$. As suggested by the model data, lightning is identified as the dominant mechanism in the regime of very large fires. 541 fires had a size of more than $300 \mathrm{~km}^{2}$ in area, and among them 516 fires had also a known cause. The man made fires accounted for only 62 events ( $11 \%$ compared to $25 \%$ in total) and the lightning fires for 454 events (84\% compared to $72 \%$ in total).

To substantiate the validity of our arguments and results, we additionally analysed the LFDB data by ecozones (Wiken, 1986). Ecozones with less than 500 fires were not taken into account, as well as subsets with less than 100 events. The results are summarised in Table 1, ordered by decreasing frequency of lightning-induced fires. 


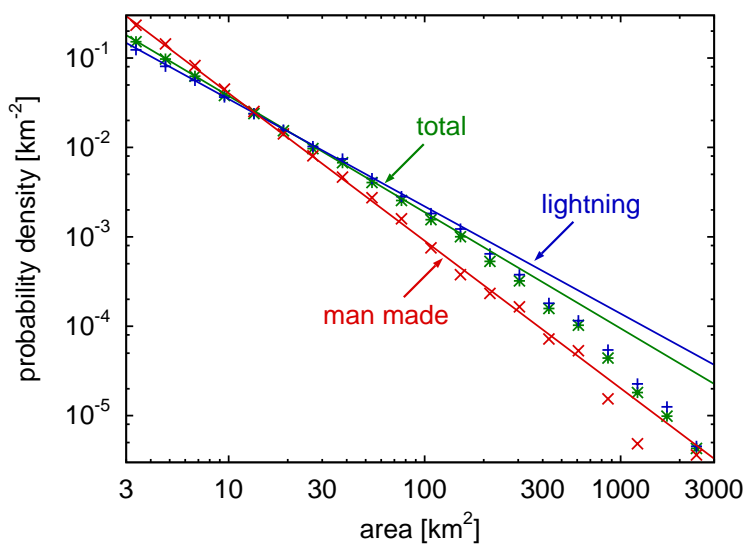

Fig. 5. Probability densities derived from the Canadian Large Fire Database (LFDB). Points represent the logarithmically binned data (6.6 bins per decade), straight lines correspond to power laws fitted by a least squares procedure.

Lightning fires (LTG) dominate in the northern regions, man made fires (MAN) occur more frequently in the more densely populated southwestern and southern areas. As expected, the scaling exponent $\alpha$ for the overall distribution of fires tends to increase with decreasing percentage of lightning fires. Except for the Montane Cordillera, the scaling exponents of the lightning fires $\alpha_{\text {LTG }}$ just vary slightly from ecozone to ecozone and are still well described by the DS-FFM. We find the scaling exponents of the man made fires $\alpha_{\text {MAN }}$ to be higher in all cases and fairly described by the AP-FFM, except for the Boreal Cordillera, but this may be due to the somewhat lower statistics. The differences between the exponents $\Delta \alpha$ amount to $0.24 \leq \Delta \alpha \leq 0.43$, in good agreement with the results obtained from the combined simulations.

Our results may also draw alternative scenarios on some recent findings in wildfire statistics of the United States (Malamud et al., 2005). In this study, Malamud et al. reported a difference in the exponents with reference to ignition source $1.12 \leq \alpha_{\text {anthropogenic }} / \alpha_{\text {lightning }} \leq 1.30$ in the eastern third (35\% by area) of the United States (for comparison: $\alpha_{\mathrm{AP}} / \alpha_{\mathrm{DS}} \approx 1.24$ and $\alpha_{\mathrm{MAN}} / \alpha_{\mathrm{LTG}} \approx 1.34$ ). This was attributed to increased landscape heterogeneity due to historic anthropogenic forest clearance because these areas are more populated. We suggest this may also be due to two different ignition mechanisms. However, most other areas had $\alpha_{\text {antropogenic }} / \alpha_{\text {lightning }} \approx 1$, at least within $\pm 2 \sigma$ error bars. Malamud et al. also found a variation in the spatial distribution of $\alpha$. Their results indicated an east-to-west gradient of higher-to-lower $\alpha$ values across the United States. The authors suggested that this gradient may have natural drivers like climate and vegetation, or may be due to increased forest fragmentation in eastern ecoregions. We propose alternatively that this gradient is an overall result of increased human activity. In their study, Malamud et al. also found that the numbers of anthropogenic vs. lightning wildfires varies as a function of ecoregion divi-
Table 1. LFDB fires by ecozones (EZ): Taiga Shield (TS), Taiga Plains (TP), Boreal Cordillera (BC), Boreal Shield (BS), Montane Cordillera (MC), and Boreal Plains (BP).

\begin{tabular}{lrrrrrrrrr}
\hline EZ & TOT & $\alpha$ & LTG & Perc. & $\alpha_{\text {LTG }}$ & MAN & Perc. & $\alpha_{\text {MAN }}$ & $\Delta \alpha$ \\
\hline TS & 1696 & 1.16 & 1579 & $93 \%$ & 1.15 & 77 & $5 \%$ & - & - \\
TP & 1211 & 1.20 & 1046 & $86 \%$ & 1.17 & 71 & $6 \%$ & - & - \\
BC & 755 & 1.18 & 586 & $78 \%$ & 1.11 & 149 & $20 \%$ & 1.35 & 0.24 \\
BS & 4150 & 1.30 & 3194 & $77 \%$ & 1.24 & 813 & $20 \%$ & 1.56 & 0.32 \\
MC & 723 & 1.60 & 329 & $46 \%$ & 1.47 & 353 & $49 \%$ & 1.74 & 0.27 \\
BP & 1724 & 1.51 & 735 & $43 \%$ & 1.27 & 952 & $55 \%$ & 1.70 & 0.43 \\
\hline CA & 11231 & 1.30 & 8086 & $72 \%$ & 1.20 & 2754 & $25 \%$ & 1.61 & 0.41 \\
\hline
\end{tabular}

sion, with 92\%-98\% anthropogenic fires in the eastern ecoregions, $17 \%-44 \%$ in the mid-level and $55 \%-82 \%$ in the remaining western ecoregions. This indicates that the observed gradient is closely related to the ratio of lightning-induced and man made fires. The bias in the western ecoregions might be due to increased fire protection activities. In the Western United States, 38\% of new home construction is adjacent to or intermixed with the wildland/urban interface (US Fire Administration, 2002). This has serious consequences. Minnich (1983, 2001) and Minnich and Chou (1997) found that the value of $\alpha$ for chaparral fires in southern California (SCA) and northern Baja California (BCA) is systematically associated with fire suppression strategies. Their analysis suggested that, as a result of different fire protection management policies in SCA (actioned) and BCA (non-actioned), the fine-grained patch structure in BCA shifted to a coarse structure in SCA, thereby reducing the number of fires and increasing fire size, but without changing the total burnt area. This has the reverse effect that the value of $\alpha$ increases with enhanced fire fighting efforts.

\section{Summary and conclusions}

In summary, we have presented a straightforward modification of the established Drossel-Schwabl forest fire model that distinguishes between lightning-induced and man made wildfires and enables a systematic increase of the scaling exponent $\alpha$ by approximately 1/3. Combined simulations of both the original and the modified model predict a dependence of the overall event-size distribution on the ratio of lightning-induced and man made forest fires as well as a difference between their respective event-size statistics. Lightning is identified as the dominant mechanism in the regime of the largest fires. The decrease of the scaling exponent $\alpha$ with lightning probability, the splitting of the partial distributions as well as the identification of lightning as the dominant mechanism in the regime of the largest fires are confirmed by the analysis of the Canadian Large Fire Database. The results also provide alternative interpretations on recent findings in wildfire statistics of the United States and suggest 
that lightning-induced and man made forest fires cannot be treated separately in wildfire modelling, hazard assessment and forest management.

Acknowledgements. This work was funded by the "Fonds zur Förderung der wissenschaftlichen Forschung (FWF)", contract no. P19733-N10.

Edited by: A. Günther

Reviewed by: two anonymous referees

\section{References}

Bak, P.: How Nature Works: The Science of Self-Organized Criticality, Springer, New York, NY, 1996.

Bak, P., Tang, C., and Wiesenfeld, K.: Self-organized criticality: An explanation of the 1/f noise, Phys. Rev. Lett., 59, 381-384, doi:10.1103/PhysRevLett.59.381, 1987.

Caldarelli, G., Frondoni, R., Gabrielli, A., Montuori, M., Retzlaff, R., and Ricotta, C.: Percolation in real wildfires, Europhys. Lett., 56, 510-516, doi:10.1209/epl/i2001-00549-4, 2001.

Canadian Forest Service: Canadian Large Fire Database (LFDB), http://cwfis.cfs.nrcan.gc.ca/en_CA/lfdb, 2002.

Cardille, J. A., Ventura, S. J., and Turner, M. G.: Environmental and social factors influencing wildfires in the Upper Midwest, United States, Ecol. Appl., 11, 111-127, doi:10.1890/1051-0761(2001) 011[0111:EASFIW]2.0.CO;2, 2001.

Clar, S., Drossel, B., and Schwabl, F.: Scaling laws and simulation results for the self-organized critical forest-fire model, Phys. Rev. E, 50, 1009-1018, doi:10.1103/PhysRevE.50.1009, 1994.

Drossel, B. and Schwabl, F.: Self-organized critical forestfire model, Phys. Rev. Lett., 69, 1629-1632, doi:10.1103/ PhysRevLett.69.1629, 1992.

Drossel, B. and Schwabl, F.: Forest-fire model with immune trees, Physica A, 199, 183-197, doi:10.1016/0378-4371(93)90001-K, 1993.

Grassberger, P.: Critical behaviour of the Drossel-Schwabl forest fire model, New J. Phys., 4, 17.1-17.15, doi:10.1088/1367-2630/ 4/1/317, 2002.

Grossman, T. and Aharony, A.: Structure and perimeters of percolation clusters, J. Phys. A: Math. Gen., 19, L745-L751, doi: 10.1088/0305-4470/19/12/009, 1986.

Gutenberg, B. and Richter, C. F.: Seismicity of the Earth and Associated Phenomena, 2nd edn., Princeton University Press, Princeton, NJ, 1954.

Hergarten, S.: Self-Organized Criticality in Earth Systems, Springer-Verlag, Berlin Heidelberg New York, 2002.

Hovius, N., Stark, C. P., and Allen, P. A.: Sediment flux from a mountain belt derived by landslide mapping, Geology, 25, 231234, doi:10.1130/0091-7613(1997)025, 1997.

Jensen, H. J.: Self-Organized Criticality - Emergent Complex Behavior in Physical and Biological Systems, Cambridge University Press, Cambridge, UK, in Cambridge Lecture Notes in Physics, no. 10, 1998.
Kunz, H. and Souillard, B.: Essential singularity in percolation problems and asymptotic behavior of cluster size distribution, J. Stat. Phys., 19, 77-106, doi:10.1007/BF01020335, 1978.

Maingi, J. K. and Henry, M.: Factors influencing wildfire occurrence and distribution in eastern Kentucky, USA, Int. J. Wildland Fire, 16, 23-33, doi:10.1071/WF06007, 2007.

Malamud, B. D., Morein, G., and Turcotte, D. L.: Forest fires: an example of self-organized critical behavior, Science, 281, 18401842, doi:10.1126/science.281.5384.1840, 1998.

Malamud, B. D., Turcotte, D. L., Guzzetti, F., and Reichenbach, P.: Landslide inventories and their statistical properties, Earth Surf. Proc. Land., 29, 687-711, doi:10.1002/esp.1064, 2004.

Malamud, B. D., Millington, J. D. A., and Perry, G. L. W.: Characterizing wildfire regimes in the United States, P. Natl. Acad. Sci. USA, 102, 4694-4699, doi:10.1073/pnas.0500880102, 2005.

Minnich, R. A.: Fire mosaics in Southern California and Northern Baja California, Science, 219, 1287-1294, doi:10.1126/science. 219.4590.1287, 1983.

Minnich, R. A.: An integrated model of two fire regimes, Conserv. Biol., 15, 1549-1553, doi:10.1046/j.1523-1739.2001.01067.x, 2001.

Minnich, R. A. and Chou, Y. H.: Wildland fire patch dynamics in the chaparral of Southern California and Northern Baja California, Int. J. Wildland Fire, 7, 221-248, doi:10.1071/WF9970221, 1997.

Pruessner, G. and Jensen, H. J.: Broken scaling in the forest-fire model, Phys. Rev. E, 65, 056707, doi:10.1103/PhysRevE.65. 056707, 2002.

Pyne, S. J., Andrews, P. L., and Laven, R. D.: Introduction To Wildland Fire, 2nd edn., John Wiley and Sons, New York, NY, 1996.

Ricotta, C., Avena, G., and Marchetti, M.: The flaming sandpile: self-organized criticality and wildfires, Ecol. Model., 119, 7377, doi:10.1016/S0304-3800(99)00057-5, 1999.

Ricotta, C., Arianoutsou, M., Díaz-Delgado, R., Duguy, B., Lloret, F., Maroudi, E., Mazzoleni, S., Moreno, J. M., Rambal, S., Vallejo, R., and Vázquez, A.: Self-organized criticality of wildfires ecologically revisited, Ecol. Model., 141, 307-311, doi: 10.1016/S0304-3800(01)00272-1, 2001.

Schenk, K., Drossel, B., and Schwabl, F.: Self-organized critical forest-fire model on large scales, Phys. Rev. E, 65, 026135, doi: 10.1103/PhysRevE.65.026135, 2002.

Song, W., Weicheng, F., Binghong, W., and Jianjun, Z.: Selforganized criticality of forest fire in China, Ecol. Model., 145, 61-68, doi:10.1016/S0304-3800(01)00383-0, 2001.

Stephens, S. L. and Ruth, L. W.: Federal forest-fire policy in the United States, Ecol. Appl., 15, 532-542, doi:10.1890/04-0545, 2005.

Stocks, B. J., Mason, J. A., Todd, J. B., Bosch, E. M., Watson, B. M., Amino, B. D., Flannigan, M. D., Hirsch, K. G., Logan, D. L., Martell, D. L., and Skinner, W. R.: Large forest fires in Canada, 1959-1997, J. Geophys. Res., 108, 8149, doi:10.1029/ 2001JD000484, 2002.

US Fire Administration: Fires in the wildland/urban interface, Topical Fire Report Series, 2, 2002.

Wiken, E. B.: Terrestrial ecozones of Canada, Lands Directorate, Environment Canada, Ottawa, Canada, Ecological land classification series, 19, 26 pp., 1986. 